\title{
The Ascending Double-Cone A Closer Look at a Familiar Demonstration
}

\author{
Sohang C. Gandhi and Costas J. Efthimiou \\ Department of Physics \\ University of Central Florida \\ Orlando, FL 32816
}

\begin{abstract}
The double-cone ascending an inclined V-rail is a common exhibit used for demonstrating concepts related to center-of-mass in introductory physics courses [1. While the conceptual explanation is well-known - the widening of the ramp allows the center of mass of the cone to drop, overbalancing the increase in altitude due to the inclination of the ramp - there remains rich physical content waiting to be extracted through deeper exploration. Such an investigations seems to be absent from the literature. This article seeks to remedy the omission.
\end{abstract}

\section{Introduction}

The, familiar, double-cone demonstration is illustrated in figure 1 bellow. The set-up consists of a ramp, inclined at an angle $\theta$, composed of two rails forming a ' $\mathrm{V}$ ' and each making an angle $\phi$ with their bisector. A double-cone of angle $2 \psi$ is placed upon the ramp. For certain values of the angles $\theta, \phi$, and $\psi$, the double-cone will spontaneously roll upward.
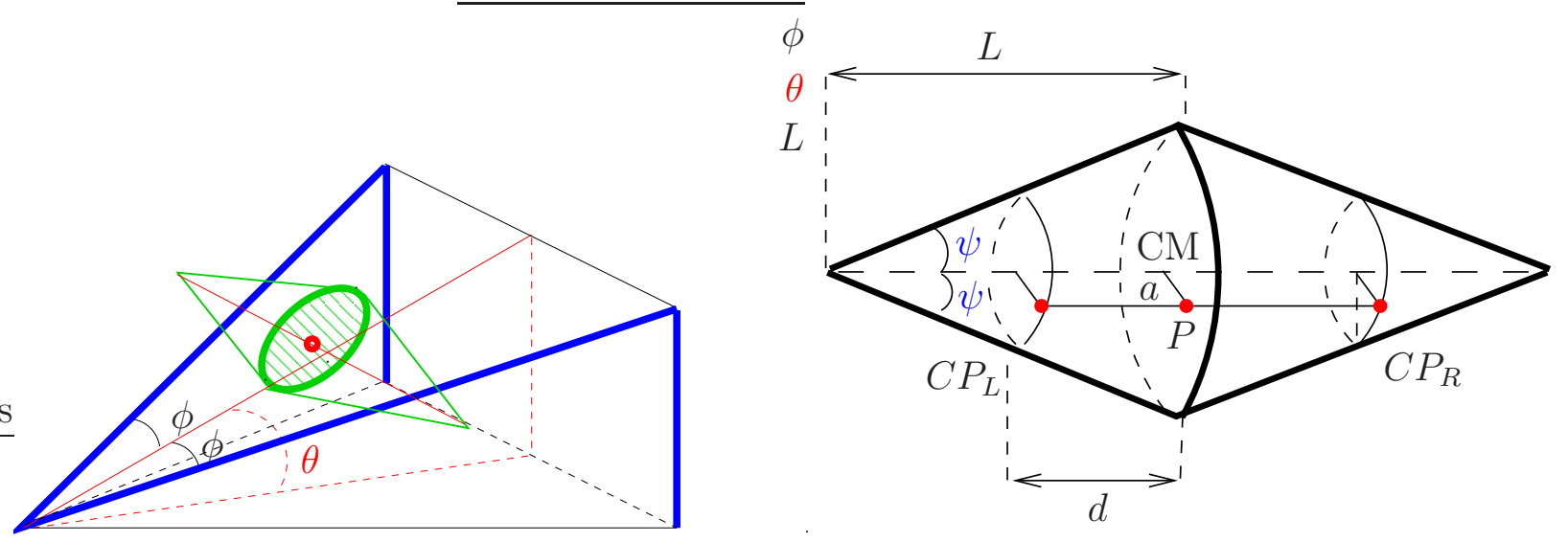

Figure 1: A double cone that rolls up a V-shaped ramp. The figure shows the principal parameters, $\theta, \phi, \psi$, determining the geometry of the demo. Also in the figure, are several quantities used in our study of the motion. In particular, $a$ is the radius of a cross-section taken at one of the contact points and perpendicularly to the axis of the cone. 
A qualitative description of the mechanism responsible for the phenomenon is easily given. As the cone moves up the ramp and gains altitude $\Delta h$, the distance between the rails increases and the contact points move nearer the symmetry axis, thus reducing the elevation of the center-of-mass (CM) relative to the ramp by an amount $\Delta H$, which is determined by (but not necessarily equal to) the change of radius $\Delta a$ resulting from the outward shifting of the contact points (figure 2). The net change of the CM height is thus $\Delta h-\Delta H$, which may be positive, negative, or zero. When it is negative the cone rolls uphill since the effect of the widening of the ramp overbalances the increase in altitude.

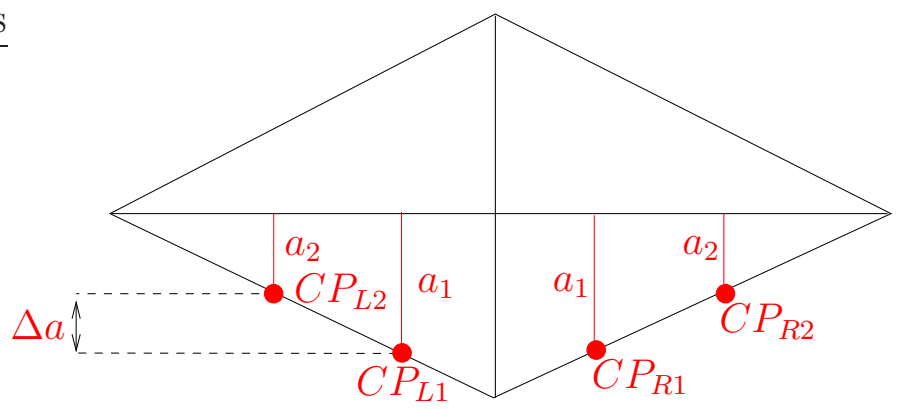

Figure 2: The shifting of the contact points as the double-cone rolls uphill. The reader is cautioned. As shall be explained, the contact points do not lie on the vertical plane that contains the symmetry axis of the cone; instead with the symmetry axis of the cone, they define a plane that intersects the vertical plane at an angle. See also figure 1

Despite the above well-known and well-understood explanation for the motion of the double cone, it seems - to the best of our knowledge - that no detailed, quantitative account has been written. Our work seeks to remedy this omission.

Towards this goal we present some notation which will facilitate the discussions in the following sections. As illustrated in the right side of figure [1] and figure 3 , we shall denote the left and right points of contact as $C P_{L}$ and $C P_{R}$ respectively, the half length of the cone as $L$, the distance between the middle cross-section of the cone and the contact points as $d$, and the distance from the axis of symmetry to the points of contact as $a$. Also, let $P$ be the point where the line defined by the two contact points intersects the middle cross-section of the cone and $\vec{a}$ be the vector that points from the center of mass (CM) of the cone to $P$. The magnitude $a$ of $\vec{a}$ is equal to the radius of the cross-section of the cone taken at the contact points perpendicularly to the axis of the cone. When the cone is at the vertex of the ramp, the two contact points coincide and $a$ has a value of $L \tan \psi$. Also, we shall denote by $\pi-\alpha$

the angle the vector $\vec{a}$ makes with the unit normal vector $\hat{K}$ to the ramp. All this is better illustrated in figure 3

\section{Net Change of Height}

To carry out our calculation we shall introduce two coordinate systems: First, a fixed system, which we shall call the ramp coordinate system $X Y Z$, with origin at the vertex of the ramp, the $Y$-axis along the bisector of the rails, the $Z$-axis perpendicular to the plane defined by the rails 


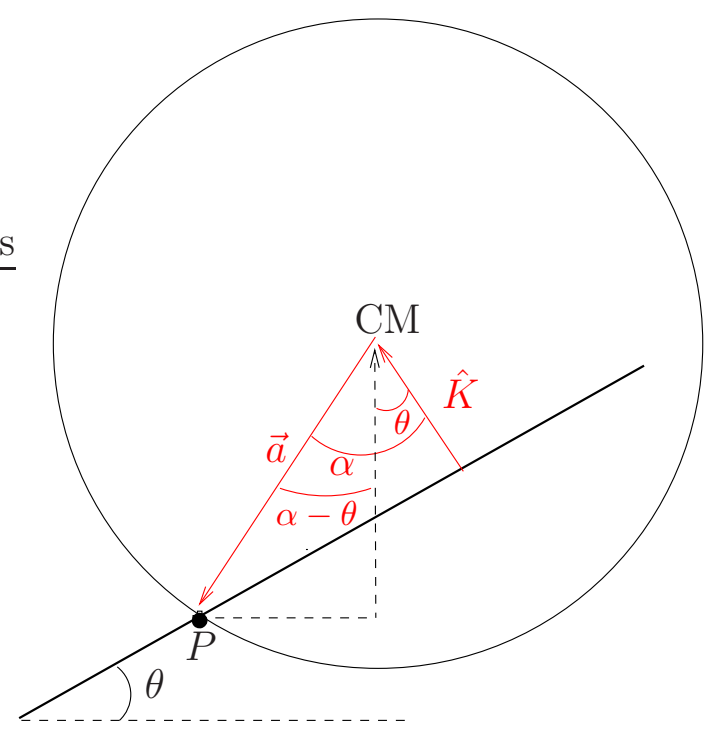

Figure 3: The circle represents part of the middle cross-section of the cone. (See right part of figure团) The solid thick straight line is the intersection of the plane defined by the two rails and a vertical plane perpendicular to the axis of the cone.

and the $X$-axis parallel to the symmetry axis of the double-cone. We also adopt a CM-system with axes parallel to those of the ramp system but whose origin is attached to the CM of the double cone. The systems are shown in figure 4

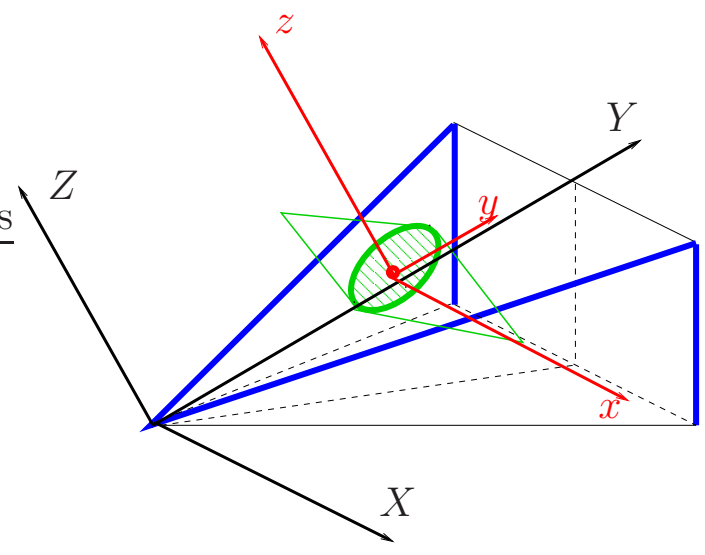

Figure 4: The ramp and CM systems.

Our experience with uniform spheres, discs, or cylinders rolling on an incline suggests that a line drawn at a contact point perpendicularly to the incline on which the objects roll passes through the CM (or the symmetry axis). Indeed, for these particular shapes, this is implied by the fact that the incline must be tangent to the surface of the object at the contact point and that the radii of a circle are normal to the circle. However, the conical shape on the $\mathrm{V}$-shaped ramp does not posses the same geometric property (although a double-cone rolling on two parallel rails would). Consequently, the contact points, $C P_{R}$ and $C P_{L}$, have a different spatial 
relation to the $\mathrm{CM}$ of the cone (figure 3).

Instead of working with the two points $C P_{R}, C P_{L}$, it is more convenient to work with the point $P$ defined previously. Notice that all three points $C P_{R}, C P_{L}, P$ have the same $z$ and $y$ coordinates and only differ in their $x$ coordinates. In fact, due to symmetry - we assume the cone to be placed symmetrically between the rails so that there is no motion in the $X$ direction - we are really dealing with a 2-dimensional problem.

To determine the angle $\alpha$ of figure 3. we must find the $y$ coordinate $y_{P}$ in the CM-frame. This will give us the location of $P$ relative to the $\mathrm{CM}$ of the cone. To this end, we write the equation

$$
z^{2}+y^{2}-\tan ^{2} \psi(L-x)^{2}=0
$$

that describes the cone and, from this, we construct the surface

$$
z=-\sqrt{(L-x)^{2} \tan ^{2} \psi-y^{2}} \equiv f(x, y)
$$

which represents the lower right quarter of the cone in the CM-frame. We then calculate the gradient of the function $f(x, y)-z$,

$$
\nabla z=\frac{1}{\sqrt{(L-x)^{2} \tan ^{2} \psi-y^{2}}}\left((L-x) \tan ^{2} \psi, y,-1\right),
$$

that is normal to the cone. From the definition of the two frames $x y z$ and $X Y Z$ we immediately see that $x=X$ and therefore $x_{C P}=X_{C P}=d$. Thus, at the right-hand contact point

$$
\left.\nabla z\right|_{C P}=\frac{1}{\sqrt{(L-d)^{2} \tan ^{2} \psi-y_{P}^{2}}}\left((L-d) \tan ^{2} \psi, y_{P},-1\right)
$$

We also construct the unit vector $\hat{u}$ whose direction coincides with the right-hand rail and points away from the vertex of the ramp. In other words, the vector $\hat{u}$ lies in the $x y$-plane and makes an angle $\phi$ with the $y$-axis; it can therefore be written as

$$
\hat{u}=(\sin \phi, \cos \phi, 0)
$$

As the cone rolls on the rails, the rails are always tangent to the cone's surface and, therefore, perpendicular to the normal $\nabla z$ :

$$
\left.\nabla z\right|_{C P} \cdot \hat{u}=0
$$

from which we locate the point $P$,

$$
y_{P}=-(L-d) \tan ^{2} \psi \tan \phi
$$

From figure 3, it can be seen that $y_{P}$ may be written in terms of $\alpha$ as

$$
y_{P}=-a \sin \alpha .
$$

Also, from figure $\$$ it can be seen that $a$ can be written

$$
a=(L-d) \tan \psi
$$



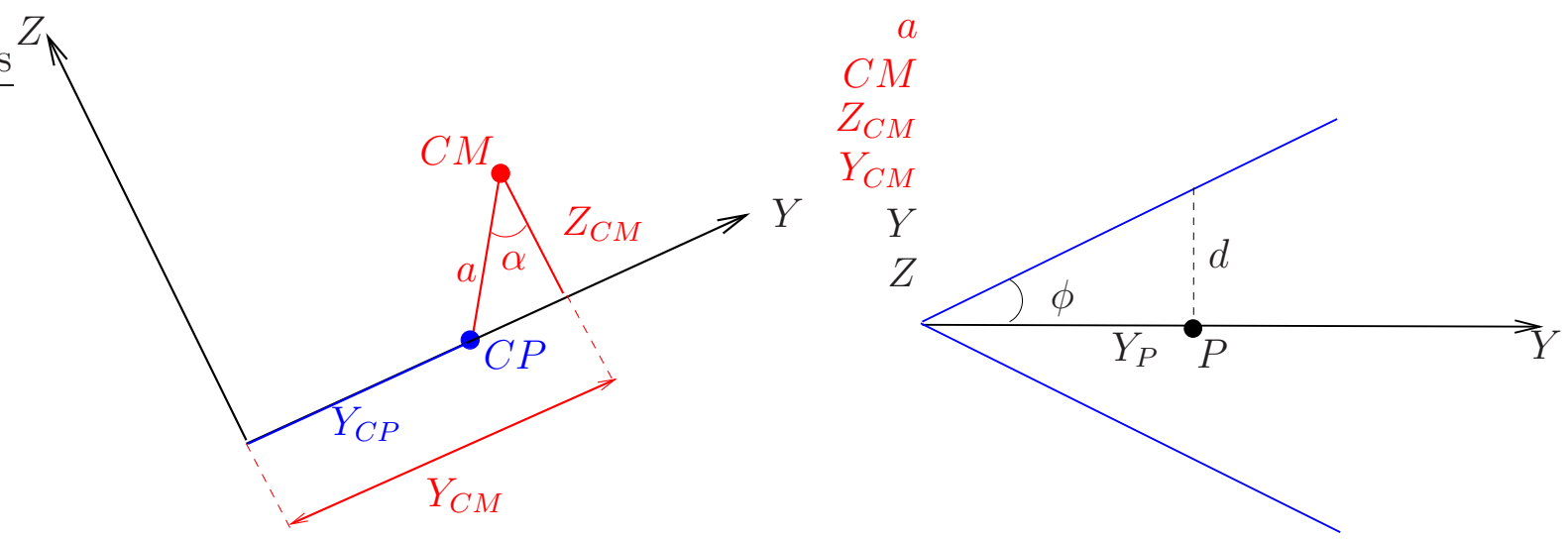

Figure 5: The relation between some of the parameters used in the study of the motion of the cone.

From equations (12), (2), (3), we thus find

$$
\sin \alpha=\tan \phi \tan \psi,
$$

which expresses the angle $\alpha$ in terms of the known angles $\phi$ and $\psi$. Since $-1 \leq \sin \alpha \leq 1$, there will be a solution for $\alpha$ if the angles $\phi$ and $\psi$ are such that $-1 \leq \tan \phi \tan \psi \leq 1$. Since $\phi, \psi$ must all lie in $\left[0, \frac{\pi}{2}\right)$, we have that $\tan \phi, \tan \psi$ are non-negative and therefore $0 \leq \tan \phi \tan \psi$. We use figure 6 to examine the other side of the inequality. In the figure, we draw a projection of the cone onto the ramp plane when $\phi=\pi / 2-\psi$. In this case, the cone exactly 'fits' the wedge created by the rails. If we increase the angle $\phi$, that is if $\phi \geq \pi / 2-\psi$, then the cone is allowed to fall through the rails. Therefore, the demo is well-made if

$$
\phi<\frac{\pi}{2}-\psi .
$$

For angles in the first quadrant, the tangent function is strictly increasing. Therefore, the previous condition implies

$$
\tan \phi<\tan \left(\frac{\pi}{2}-\psi\right)=\cot \psi=\frac{1}{\tan \psi} \Rightarrow \tan \phi \tan \psi<1 .
$$

Therefore, for a well-made demo, the inequality

$$
0 \leq \tan \phi \tan \psi<1
$$

is always satisfied and the angle $\alpha$ has a well-defined value.

We are now able to see the reasoning behind the statement $\Delta a \neq \Delta H$ made at the introduction. The angle between $\vec{a}$ and the vertical is $\alpha-\theta$ (figure 3 ). Thus, the change in the height $\Delta H$ of the CM relative to the points of contact is in fact given by $\Delta a \cos (\alpha-\theta)$. It follows that the net change in height of the $\mathrm{CM}$ is

$$
\text { net change of height }=\Delta h-\Delta a \cos (\alpha-\theta) \text {. }
$$




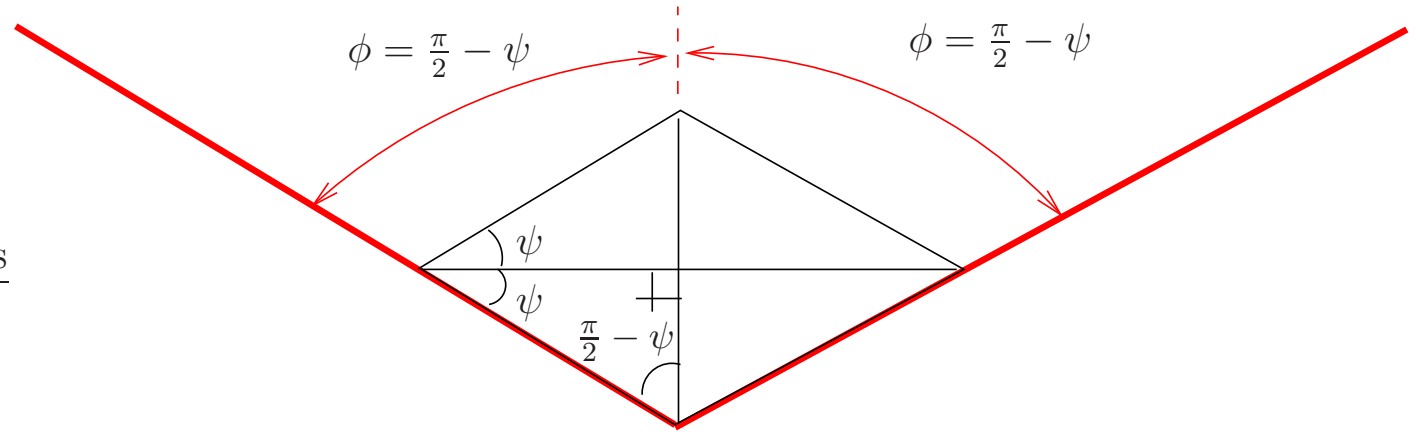

Figure 6: When a cone of angle $\psi=\frac{\pi}{2}-\phi$, is projected on the ramp plane, then the cone exactly 'fills' the wedge formed by the rails. Increasing the angle $\phi$ implies a wedge bigger than what the cone can 'fill'.

\section{A Dynamical Explanation}

With our newfound knowledge regarding the way in which the double-cone sits on the ramp, we are in a position to provide an account from a dynamical perspective.

Any solid placed on the ramp will contact it at two points. Since we assume that the solid rolls without slipping, the contact points must be instantaneously at rest. The rigidity of the solid then implies that all points lying on the line connecting the two contact points are also at rest instantaneously, and, hence, this line defines the instantaneous axis of rotation. The torque about this axis will solely be due to gravity since the frictional forces and the reaction forces of the ramp are exerted at the axis.

The situation for a cylinder placed on the ramp is shown in figure 7 The angle between the weight and the vector $\vec{a}$, which enters the torque, is determined by the inclination $\theta$ of the ramp only. Thus the torque resultant of the weight, $-m g a \sin \theta$, always tends the cylinder to roll down the ramp.

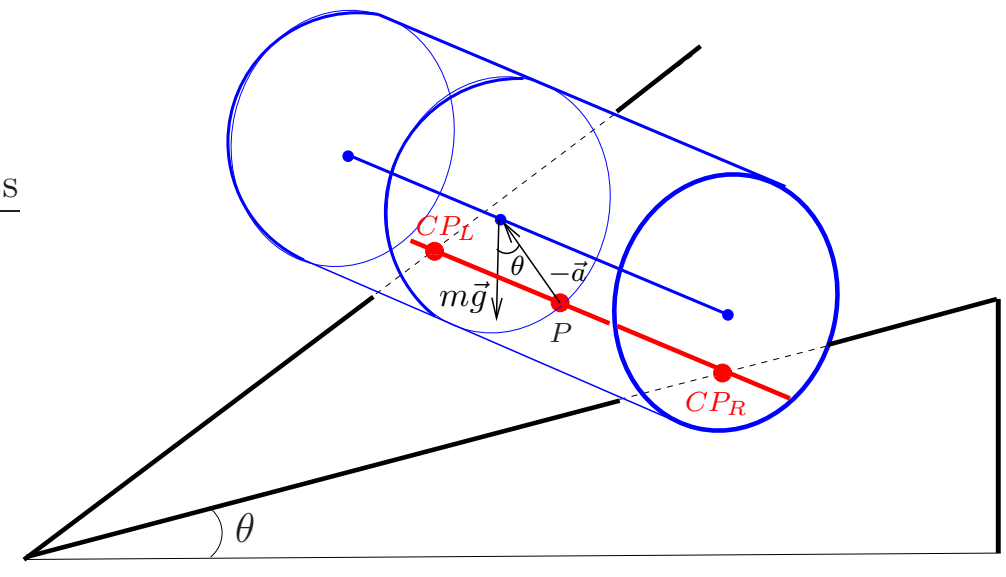

Figure 7: The resultant torque always pulls a cylinder down the ramp.

The situation for the double-cone is shown in figure [8. The angle between the weight and the vector $\vec{a}$ which enters the torque is now $\pi-(\alpha-\theta)$. The torque resultant of the 
weight, $m g a \sin (\alpha-\theta)$, depends strongly on the value of $\alpha$, which can change by changing the parameters $\phi$ and $\psi$. If $\alpha>\theta$, the resultant torque will tend the solid up the ramp. We can thus see that it is the unusual way in which the double-cone sits on the ramp that distinguishes it from the other solids and provides it the mechanism with which to roll up the ramp.

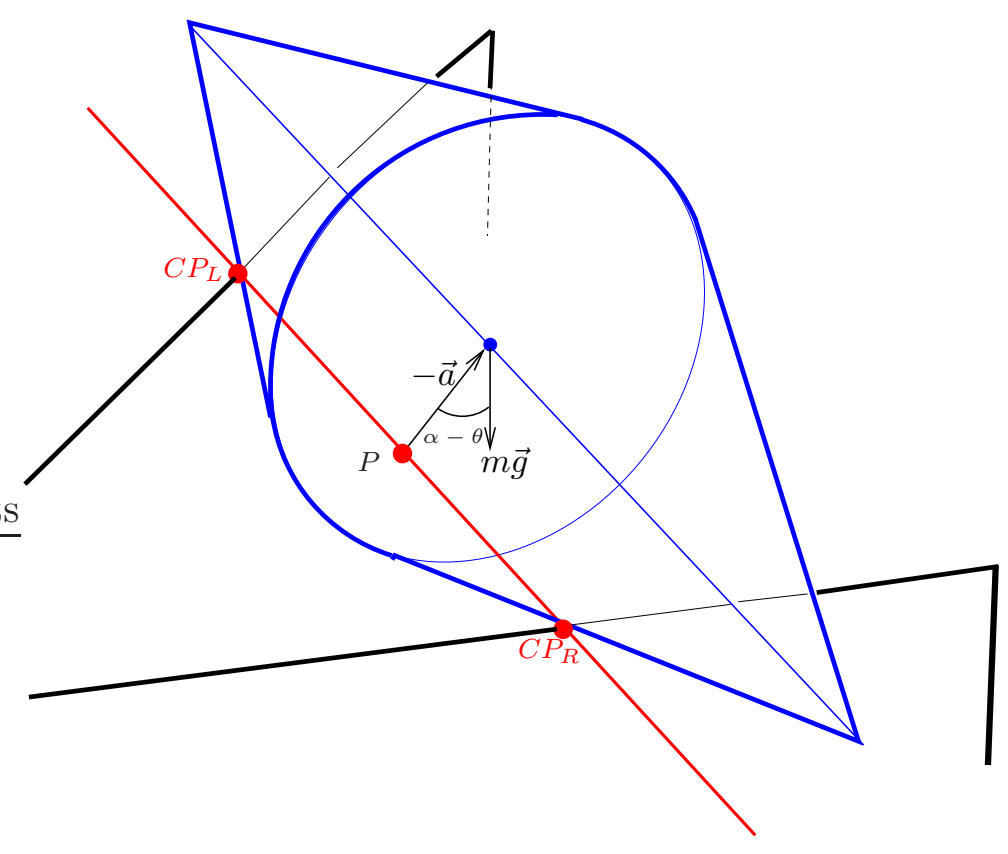

Figure 8: The resultant torque in the case of a double cone can be positive, negative, or zero depending on the value of $\alpha$.

\section{The Motion of the CM}

Since $Z$ is oriented perpendicular to the plane defined by the rails and $P$ lies on the ramp plane, $Z_{P}$ is always zero. Thus $Z_{C M}$ is given by

$$
Z_{C M}=a \cos \alpha .
$$

(See figure 5) If the point $P$ has coordinate $Y_{P}$ in the ramp-frame, then the half-distance between the rails, $d$, must be given by

$$
d=Y_{P} \tan \phi,
$$

as is evident from figure [5 Using equations (6), (3), and (4), we write for $a$,

$$
a=L \tan \psi-Y_{P} \sin \alpha .
$$

Hence,

$$
Z_{C M}=\left(L \tan \psi-Y_{P} \sin \alpha\right) \cos \alpha .
$$


Referring again to figure 5, we obtain

$$
Y_{C M}=Y_{P}+a \sin \alpha=Y_{C P}+\left(L \tan \psi-Y_{C P} \sin \alpha\right) \sin \alpha,
$$

or

$$
Y_{P}=\frac{Y_{C M}-L \tan \psi \sin \alpha}{\cos ^{2} \alpha} .
$$

Plugging this in to our previous result we obtain

$$
Z_{C M}=\lambda Y_{C M}+b,
$$

where $\lambda$ and $b$ are constants determined by the geometry of the demo:

$$
\lambda=-\tan \alpha, \quad b=L \tan \psi(\cos \alpha-\tan \alpha \sin \alpha) .
$$

Equation (9) implies that the CM is constrained to move along a line on the $z y$-plane with slope $\lambda$ and intercept $b$.

This result is also made apparent through physical reasoning. The cone's motion is that of rigid rotation about the symmetry axis plus a translational motion of the CM with velocity $\vec{v}_{C M}$. Thus, the total velocity of the point $P$ is $\vec{v}_{r o t, P}+\vec{v}_{C M}$, where $\vec{v}_{r o t, P}$ is its rotational velocity about the symmetry axis. The rolling without slipping condition implies that the velocity of the point $P$ must be zero. Therefore

$$
\vec{v}_{C M}=-\vec{v}_{r o t, P}
$$

Since $\vec{v}_{\text {rot }, P}$ must be perpendicular to $\vec{a}$, we conclude that

$$
\vec{v}_{C M} \perp \vec{a}
$$

Since $\vec{a}$ is fixed in direction and the velocity of the CM must always lie on the $Y Z$-plane (by symmetry), the CM's path is a line declined (inclined if $\alpha<\theta$ ) below the horizontal by an angle $\alpha-\theta$ (see figure 9).

\section{$5 \quad$ Energy Analysis}

As we have seen, the path of the CM is a straight line that makes an angle $\alpha$ with $Y$-axis as shown in figure 9. We now introduce a new generalized coordinate: the distance along this line, $q$. We shall take its zero to correspond to the position of the CM when the cone is at the bottom of the ramp. In figure 9, $\vec{a}_{0}$ represents the value of $\vec{a}$ when the cone is at the bottom of the ramp and $d=0$. It can be seen that, in terms of $q, Y_{C M}$ is given by

$$
Y_{C M}=Y_{C M}^{0}+q \cos \alpha
$$

where $Y_{C M}^{0}$ is the coordinate of the $\mathrm{CM}$ when the cone is at the bottom of the ramp- that is when $q=0$. It is also clear from the figure that

$$
Y_{C M}^{0}=a_{0} \sin \alpha=L \tan \psi \sin \alpha .
$$



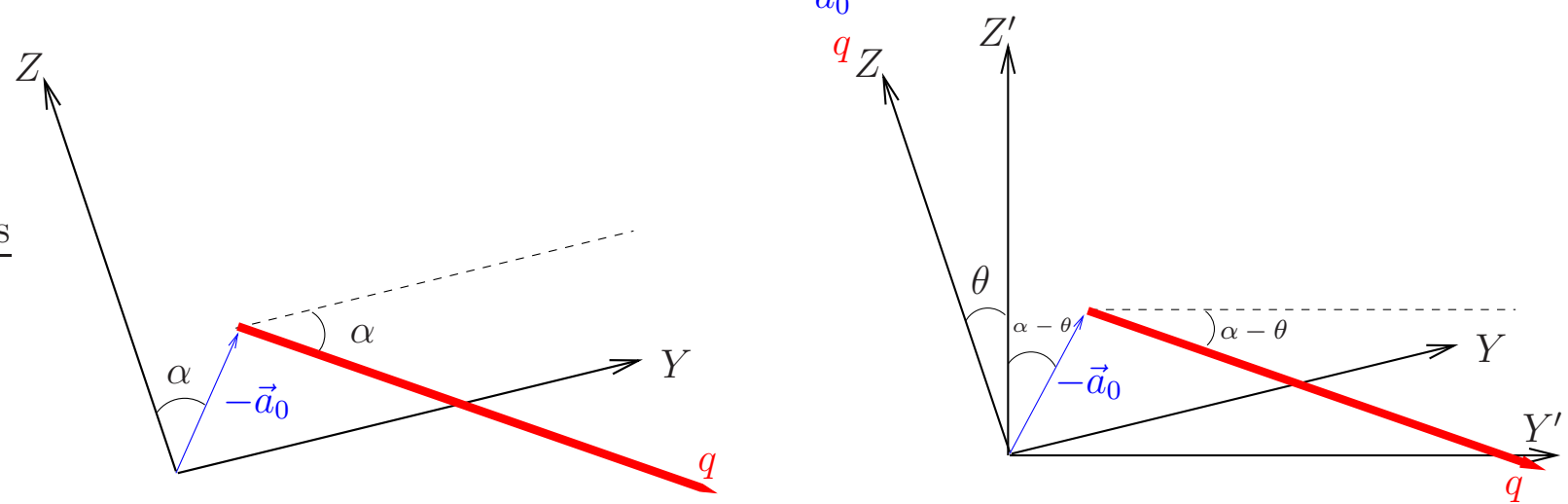

Figure 9: The motion of the CM in the $X Y Z$ frame and in the $X^{\prime} Y^{\prime} Z^{\prime}$ frame - a clockwise rotation of $X Y Z$ about the $X$-axis by $\theta$. The thick straight line represents the trajectory of the CM.

Combining the results with equations (17) and (8), we obtain

$$
a=L \tan \psi-q \tan \alpha .
$$

The rolling without slipping constraint implies that the angular velocity $\omega$ of the cone about the symmetry axis is given by

$$
\omega=-\frac{\dot{q}}{a}=-\frac{\dot{q}}{L \tan \psi-q \tan \alpha} .
$$

Thus, we obtain for the kinetic energy

$$
T=\frac{1}{2} m \dot{q}^{2}+\frac{1}{2} I \omega^{2}=\frac{1}{2}\left[m+\frac{I}{(L \tan \psi-q \tan \alpha)^{2}}\right] \dot{q}^{2},
$$

where $I$ is the moment of inertia of the cone with respect the symmetry axis.

For the potential energy, we are concerned with the vertical height of the CM; that is, we must find the coordinate $Z_{C M}^{\prime}$ in terms of $q$, where the primed frame $X^{\prime} Y^{\prime} Z^{\prime}$ is one that is obtained from the unprimed frame $X Y Z$ by rotating the latter by an angle $\theta$ about the $X$-axis (see figure 9). From the figure we see that

$$
Z_{C M}^{\prime}=Z_{C M}^{\prime 0}-q \sin (\alpha-\theta) .
$$

Thus, we have for the potential

$$
U=-m g q \sin (\alpha-\theta),
$$

where we have set the potential to zero at the initial position of the cone.

For the cone to roll up the ramp, the potential must be a decreasing function of $q$, that is

$$
\sin (\alpha-\theta)>0 .
$$

With the use of equation (4) and some trigonometric identities, this condition may equivalently be written

$$
\tan \theta<\frac{\tan \phi \tan \psi}{\sqrt{1-\tan ^{2} \phi \tan ^{2} \psi}} .
$$


Recalling the inequality (5), we see the denominator is well-defined and is not singular or imaginary.

We may now write down the energy:

$$
E=T+U=\frac{1}{2}\left[m+\frac{I}{(L \tan \psi-q \tan \alpha)^{2}}\right] \dot{q}^{2}-m g q \sin (\alpha-\theta) .
$$

It should be noted that $q$ may not take on arbitrary values. Indeed, as we have defined it, $q$ has a minimum value of 0 . There will also be a maximum value at which point the cone falls through the rails. This point corresponds to the double cone contacting the ramp at its very tips. Hence $a=0$ and, from equation (10),

$$
0 \leq q \leq L \frac{\tan \psi}{\tan \alpha}
$$

In the next section we shall see an unexpected property of the motion about the fall-through point, $q=L \tan \psi / \tan \alpha$.

\section{A Surprising Feature}

The reader should note the position dependent coefficient in the inertial term of our expression for energy (12). It is this term that gives the system unique behavior beyond what has been discussed so far.

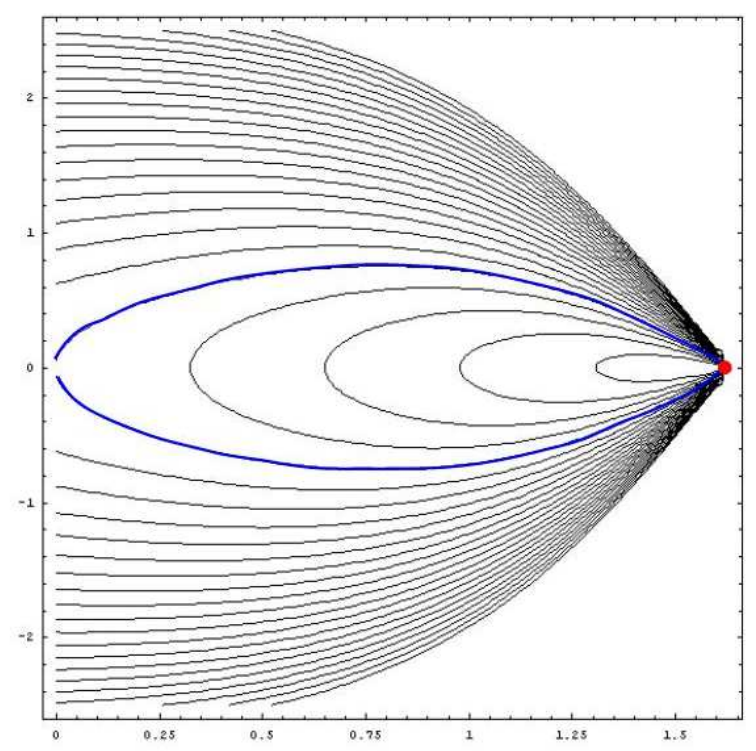

Figure 10: $\dot{q}$ vs. $q$ phase portrait for $\phi=\psi=\frac{\pi}{6}, \theta=\frac{\pi}{12}, L=m=1$.

Figure 10 shows the $\dot{q}$ vs. $q$ phase portrait for the system with $\phi=\psi=\pi / 6, \theta=\pi / 12$, and $L=m=1$. The first astonishing fact is that all of the curves go through the same point. This is the fall-through point and it will be the subject a more exhaustive discussion below. 
Shown in figure 10 as a thick curve is the zero energy trajectory. Those curves contained within it correspond to progressively more negative energies. Those exterior, progressively more positive. One may be tempted to identify, as cycles, the negative energy trajectories. However, the fall-through point is a singularity. While all the trajectories approach it arbitrarily as $t \rightarrow+\infty$, none of them actually reach it. Thus, the negative energy curves are all open by one point. Their 'open loop' shape merely illustrates the simple fact that, when the cone is placed at the top of the ramp and given an initial shove, the cone will roll down for a bit and then roll back up.

To illustrate just what it is about the motion that is so startling, let's focus on a single trajectory. Consider the $E=0$ energy curve corresponding to releasing the cone from rest at the bottom of the ramp (as the demonstration is typically performed). This predicts that, at first, the cone's translational speed will be increasing reaching maximum value at about midway along its path after which the translational speed will begin to ebb, decreasing to zero at the fall-through point. The cone will indefinitely approach but never reach the fall-through point. Though, at first, hard to accept, with a little thought, one may see that this behavior does in fact make physical sense.

To make things more concrete let us divide the path of the CM in to equal tiny increments of, $\Delta q=\epsilon$. Since the potential is linear, the cone will gain a fixed amount of energy, $\delta$, over each increment. Also, since $a$ is a linear function of $q$, it will decrease by a fixed amount, $\Delta a$, over each increment.

Now, let us for the moment, consider a simplified situation; namely, we shall adjust the inclination of the ramp so that the system is potentially neutral and have a physicist take over gravity's job. We shall stipulate:

1. the cone will be given an initial speed $v$;

2. the physicist will provide and only enough energy over each increment so as to keep the cone translating as speed $v$;

3. the physicist shall not give the cone more than $\delta$ energy per increment.

From the rolling without slipping constraint we may write the cone's rotational energy as $T_{\text {rot }}=\frac{1}{2} I \frac{v^{2}}{a^{2}}$. Differentiating we obtain

$$
\Delta T_{\text {rot }}=\frac{1}{2} I \frac{v^{2}}{a^{3}} \Delta a
$$

for the amount of energy needed over each increment to keep the cone translating at speed $v$. Since $a$ goes from a maximum to zero, we can always find a position along the ramp for which this amount exceeds $\delta$. At this point, the physicist will fail to maintain the cone at its current translational speed. However, the physicist may transfer some of the translational energy into rotation, causing the cone to slow down and repeat the process for each of the following intervals.

Returning to the actual situation, with the cone rolling freely on the ramp, it is the same principle that is responsible for the cone's peculiar motion. However, the accountant that keeps track of energy transactions is no longer the physicist but gravity. Also, in this case, energy is initially being proportioned between the translational and rotational energy. We may for 
the sake of argument, use the speed of the cone, say, one microsecond after release, as the $v$ in equation (13). No matter how small it is, we may still find a position for which the right hand side of equation (13) exceeds $\delta$. In reality the initial increase of the speed of the cone as it moves along only serves to bring about the cross-over point sooner. As seen in figure 10, the higher the energy, the more the curve 'flattens' at the beginning, eventually loosing the increasing part altogether.

By straightforward extension of the above argument, we thus, see more and more energy has to be transferred form translation to rotation as $a$ shrinks down to zero. This results in an asymptotic behavior of pure rotation about the symmetry axis. The energy transfer mechanism

in all of this is ultimately friction - which we assume to be ideal-working to keep the cone from slipping.

\section{$7 \quad$ Integration of the Equation of Motion}

Equation (12) can be solved for the velocity,

$$
\dot{q}= \pm \sqrt{\frac{2(E+m g q \sin (\alpha-\theta))}{m+\frac{I}{(L \tan \psi-q \tan \alpha)^{2}}}}
$$

Notice that, except for in the degenerate cases in which $\phi$ or $\psi=0$ (and thus also $\alpha=0$ ),

$$
\lim _{q \rightarrow L \frac{\operatorname{tan\psi }}{\tan \alpha}} \dot{q}=0
$$

Thus the ebb in the cones speed is a general feature.

Equation (14) gives the velocity of the CM as a function of position, $\dot{q}=\dot{q}(q)$. We can find where the cross-over point occurs by taking the derivative of this equation and setting it equal to zero. The resulting expression is quite turbid. More meaningful is a plot of the cross-over point versus energy. This is shown in figure 11 where we used the same values of the parameters used for the phase plot of figure 10.

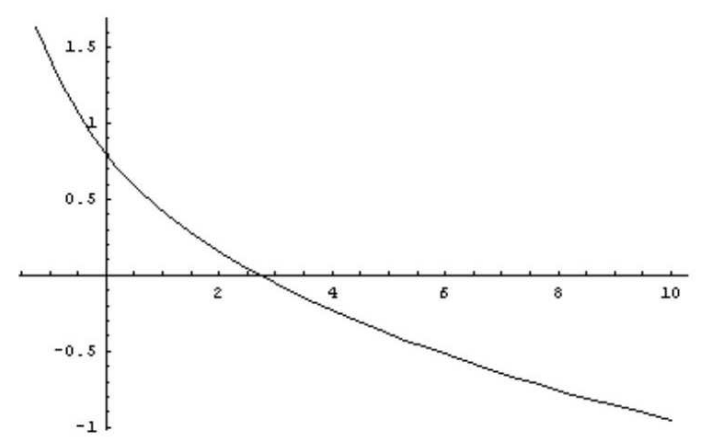

Figure 11: Cross-over point vs. Energy 
Notice that the cross-over point is a decreasing function of energy. As we have already pointed out, increasing energy means increasing the starting velocity which brings the crossover point closer. Also interesting is that, above a certain energy, the cross-over point occurs at negative values of $q$. For large enough energy, the initial velocity is so high that gravity can never provide enough energy over an increment to keep the cone translating at its current speed. Therefore at these energies the speed will be a strictly decreasing function of time. This may be quickly confirmed using the extreme case $E \rightarrow+\infty$ in (14).

We may now integrate equation (14) to get

$$
\beta \pm t=\int \sqrt{\frac{m+\frac{I}{(L \tan \psi-q \tan \alpha)^{2}}}{2(E+m g q \sin (\alpha-\theta))}} d q
$$

where $\beta$ is a constant of integration. There is no simple analytical solution to this integral. We may however make approximations in two regimes: small values of $q$ (cone close to the vertex) and values of $q$ close to $L \tan \psi / \tan \alpha$ (cone close to the fall-through point).

\subsubsection{Near Vertex Regime}

For small values of $q$, we may apply the binomial expansion to the $\frac{I}{(L \tan \psi-q \tan \alpha)^{2}}$ term in the numerator of (14) and approximate the integral as

$$
\beta \pm t \approx \int \sqrt{\frac{A+B q}{E+C q}} \frac{d q}{2}
$$

where

$$
A \equiv m+\frac{I}{L^{2} \tan ^{2} \psi}, \quad B \equiv \frac{2 I \tan \alpha}{L^{3} \tan ^{3} \psi}, \quad C \equiv m g \sin (\alpha-\theta)
$$

The last integral is elementary and gives

$$
\begin{aligned}
\beta \pm t \approx & \frac{\sqrt{\frac{A+B q}{E+C q}}(E+C q)}{C} \\
+ & \frac{(A C-B E) \sqrt{\frac{A+B q}{E+C q}} \sqrt{E+C q} \ln \left(2 \sqrt{(A+B q)(E+C q)}+\frac{B E+A C+2 B C q}{\sqrt{B C}}\right)}{2 C^{3 / 2} \sqrt{B(A+B q)}} .
\end{aligned}
$$

Figure 12 plots the solution for the example that we have been using throughout, with initial conditions $q=0$ and $\dot{q}=0$ at $t=0$. The inverse curve $q=q(t)$, is found by reflection with respect to the diagonal $q=t$ of the unit 'square'. Notice that the figure indicates that the cone is speeding up.

If one makes an additional binomial expansion on equation (15), a simpler expression is obtained

$$
q(t) \approx \frac{e^{\sqrt{E(\beta \pm t)}}\left(B^{2}-4 A E-2 B \sqrt{E} e^{\sqrt{E(\beta \pm t)}}+E e^{2 \sqrt{E(\beta \pm t)}}\right.}{4 E^{3 / 2}} .
$$




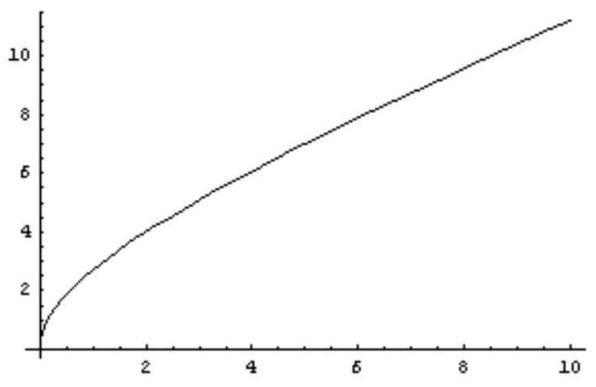

Figure 12: The graph of $t$ vs $q$ close to the vertex of the ramp.

\subsubsection{Near Fall-Through Regime}

The integral of equation (15) may be written in terms of $a$ as

$$
-\beta \pm t=\int \cot \alpha \sqrt{\frac{m+\frac{I}{a^{2}}}{D-F a}} d a,
$$

where

$$
D \equiv 2 E+2 m g L \frac{\sin (\alpha-\theta) \tan \psi}{\tan \alpha}, \quad F \equiv 2 m g \sin (\alpha-\theta) \cot \alpha \text {. }
$$

For, $q \approx L \frac{\tan \psi}{\tan \alpha} \Rightarrow a \approx 0$,

$$
-\beta \pm t \approx \sqrt{\frac{I}{D}} \cot \alpha \int \frac{d a}{a}
$$

Integrating and inverting, we obtain

$$
q(t) \approx L \frac{\tan \psi}{\tan \alpha}-\cot \alpha \exp \left[\tan \alpha \sqrt{\frac{D}{I}}(-\beta \pm t)\right]
$$
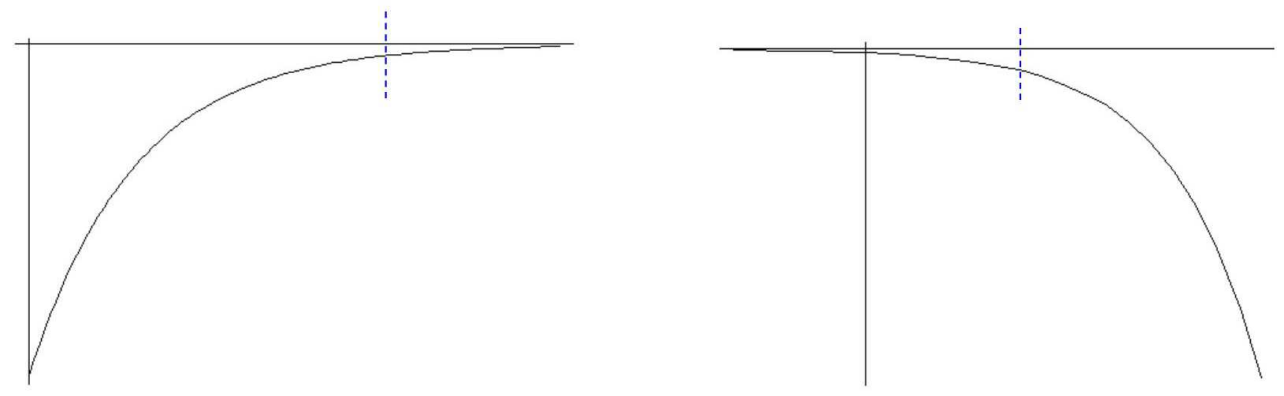

Figure 13: $q$ vs $t$ near the fall-trough point. 
The general form of the solution is that of the left side of figure 13, when the minus sign is chosen in the above expression, representing the cone moving up the ramp toward the fall through point. It is that of the right side figure 13] when the positive sign is chosen, representing the cone moving down the ramp, starting at some point very close to the fall through point at $t=0$. Notice that the graph on the left depicts the cone speeding up as it gains potential and that on the right slowing down as it drops in potential. In the figures, the dotted vertical lines roughly mark off the regions in which the solutions are valid. The vertical axes are at $q=L \frac{\tan \psi}{\tan \alpha}$, the point at which the cone falls through the rails. Due to this asymptotic behavior, it would take infinite time for the cone to reach the fall-through point or reach any other point starting from the fall-through point.

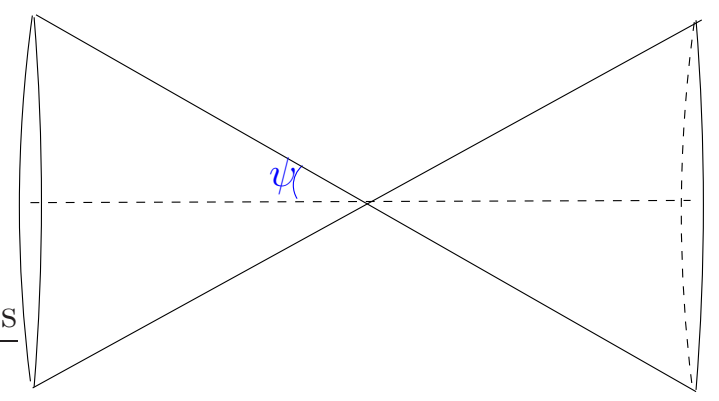

Figure 14: The simplest variation of the double-cone demo would be an hourglass sitting on a V-shaped ramp that has its vertex elevated.

\section{The Hourglass}

It is not difficult to invent variations on this system (see, for example [2]). In particular, one may obtain an essentially identical motion by 'inverting' the double-cone as well as our rampthat is, flipping the ramp up side down so that its vertex is at the top, and placing upon it an hourglass-shaped object such as the one depicted in figure 14. If we define our parameters in a similar manner as before (adjusting, as in the figure, the definition of $\psi$ appropriately), and redefine $q$ so that its zero now corresponds to the location of the CM when the hourglass is at the top of the ramp and so that it increases in the direction in which the cone travels down the ramp, we obtain for the energy,

$$
E=\frac{1}{2}\left[m+\frac{I}{q^{2} \sec ^{4} \alpha \tan ^{2} \alpha}\right] \dot{q}^{2}+m g q \sin (\alpha-\theta) .
$$

The result can be seen to posses essentially the same form as the energy of the double cone, and thus the system's motion will be similar, with the cone, now, tending to roll in the direction of decreasing $q$-again, up the ramp-and the singularity, now, occurring at $q=0$.

\section{Concluding Remarks}

We see that the implications of the unique geometry of this system extend far beyond the simple conceptual explanation relying on a rough trend of the CM. Despite the simplicity of the demo, 
the dynamical explanation, in terms of the consequences of the cone's peculiar way of sitting on the ramp, has revealed surprising properties. We hope that instructors of introductory physics will be as charmed as we are by this demo and its rich physical content.

\section{Acknowledgements}

This research was sponsored by generous grants from the University of Central Florida Honors College and Office of Undergraduate Studies. S.G. would like to thank Rick Schell and Alvin Wang for this financial support. S.G. would like to thank C.E. for the opportunity to work on this project, his generous time and guidance throughout this work and S.G.'s studies.

\section{References}

[1] PIRA demonstration 1J11.50. See http://www.physics.ncsu.edu/pira/.

[2] Nuri Balta, New versions of the Rolling Double Cone, Physics Teacher 40 (2002) 156. 